\title{
Experiência sobre o uso de ferramentas de apoio à pesquisa experimental em uma disciplina de IHC
}

\author{
Ana Paula Juliana Perin ${ }^{1}$, Polianna S. Paim ${ }^{1}$ e Natasha Malveira C. Valentim ${ }^{1}$ \\ ${ }^{1}$ Universidade Federal do Paraná (UFPR)) \\ ${ }^{1}$ Curitiba, PR, Brasil \\ ${ }^{1}$ Departamento de Informática \\ apjperin@inf.ufpr.br, pspaimeinf.ufpr.br, natasha@inf.ufpr.br
}

\begin{abstract}
The use of tools that support experimental research allows students to develop and exercise content in a more practical way. This paper presents an experience report on the use of tools to support the different stages of studies proposed in a remote discipline of Experimental Human-Computer Interaction (HCI). Thirteen undergraduate and graduate students from the Federal University of Parana (UFPR) provided feedback on the use of different tools at the end of the course. Data were analyzed quantitatively and qualitatively. Through these analyses, it was possible to understand that the tools allow students to know and have practical research experiences.
\end{abstract}

Resumo. $O$ uso de ferramentas que apoiam a realização de pesquisa experimental permite que estudantes desenvolvam e exercitem conteúdos de maneira mais prática. Esse artigo apresenta um relato de experiência sobre o uso de ferramentas como apoio às diferentes etapas de estudos propostos em uma disciplina remota de Interação Humano-Computador (IHC) Experimental. Treze estudantes de graduação e pós-graduação da Universidade Federal do Paraná (UFPR) forneceram feedback sobre o uso de diferentes ferramentas ao final da disciplina. Os dados foram analisados de forma quantitativa e qualitativa. Através destas análises foi possível entender que as ferramentas possibilitam que estudantes conheçam e tenham experiências práticas de pesquisa.

\section{Introdução}

A pesquisa experimental possibilita que futuros pesquisadores e profissionais aprendam a planejar, executar e analisar estudos que fazem parte do processo de descobertas científicas [Lazar et al. 2017]. [Kitchenham and Charters 2007] descrevem que existem dois tipos de estudos, os primários e secundários. O estudo primário é um estudo empírico que investiga uma questão de pesquisa específica. Já o estudo secundário é um estudo que revisa todos os estudos primários relacionados a uma questão de pesquisa específica com o objetivo de sintetizar evidências. Desse modo, o uso de ferramentas adequadas para apoiar as diferentes etapas destes estudos pode fornecer subsídios para que os estudantes desenvolvam e exercitem a capacidade de análise crítica e tomada de decisão sobre o seu tema de pesquisa. Além disso, o uso de ferramentas podem apoiar a autonomia no trabalho individual e em equipe, e as habilidades de comunicação oral e escrita dos pesquisadores. 
Desse modo, alguns autores como [Firmo et al. 2014] e [Pereira 2013] relatam a importância do uso de ferramentas que apoiem os processos de ensino e aprendizagem na disciplina de Interação Humano-Computador (IHC). O uso de ferramentas e a condução de práticas de IHC possibilita que estudantes exercitem o papel de tomada de decisões em suas atividades, gerando ideias e as desenvolvendo no decorrer das aulas, enquanto docentes atuem na orientação e coordenação [Firmo et al. 2014]. Nessa mesma perspectiva, [Pereira 2013] relata que estudantes de IHC, por meio de uma avaliação, combinaram teoria e prática no processo de aprendizagem e utilizaram algumas ferramentas apresentadas na disciplina, tais como Cacoo (mockups) e a CogTool (modelagem de tarefas). Para [Diniz et al. 2020] é possível engajar estudantes a aprenderem sobre o uso das ferramentas e a vivenciarem na prática em atividades profissionais.

Portanto, este artigo relata o uso de ferramentas como apoio às diferentes etapas de estudos propostos em uma disciplina de IHC Experimental. Os tópicos abordados na disciplina foram: 1) Introdução à IHC Experimental; 2) Estudos Secundários (Mapeamento e Revisão Sistemática da Literatura - MSL/RSL); 3) Estudos Primários (Experimentos Controlados); 4) Análise Quantitativa de Estudos Primários; e 5) Análise Qualitativa de Estudos Primários. Para os tópicos 2 a 5 foram sugeridas ferramentas para apoiar a execução dos trabalhos propostos, porém, os alunos tinham a liberdade de escolher outras ferramentas para serem utilizadas. Participaram desta disciplina, 2 alunos de graduação em Ciência de Computação, 1 aluna de Informática Biomédica, 6 alunos de mestrado e 4 alunos de doutorado em Informática da Universidade Federal do Paraná. O feedback sobre o uso das ferramentas adotadas na disciplina foi fornecido através de um questionário disponibilizado no Google Forms. Os dados qualitativos e quantitativos coletados foram analisados individualmente e revisados por três pesquisadoras.

A dinâmica utilizada na disciplina foi a Sala de Aula Invertida conforme descrito por [Valente 2014], que consiste no estudo do conteúdo pelo estudante antes da aula. Assim, este aluno vai para a aula mais preparado para discussões e atividades práticas, são incentivados a serem protagonistas do seu aprendizado, buscando conhecer e usar as ferramentas para a execução das atividades propostas. A disciplina foi ministrada de forma remota, contendo atividades síncronas e assíncronas, e utilizou-se o Moodle para entrega dos trabalhos, disponibilização de materiais e como canal de comunicação entre professora, monitoras e alunos.

Esse trabalho está organizado em cinco seções. A Seção 2 aborda os trabalhos relacionados. A Seção 3 trata sobre a metodologia conduzida na disciplina de IHC experimental. A Seção 4 aborda sobre a análise quantitativa e a Seção 5 sobre a análise qualitativa e discussões. Por fim, a Seção 6 aborda sobre as considerações finais e trabalhos futuros.

\section{Trabalhos Relacionados}

Nessa seção serão apresentados alguns exemplos de estudos relacionados ao uso de ferramentas como apoio aos processos de ensino e aprendizagem em disciplinas de IHC.

[Pereira 2013] relata um estudo realizado na disciplina de IHC, conduzida em um curso de Especialização em Engenharia de Software na Universidade Estadual de Campinas (Unicamp). Nesse estudo, foram realizadas seis aulas intercalando entre conteúdos teóricos e práticos. Os conteúdos abordados nesse estudo foram: introdução à IHC, pro- 
cesso de design (Design Socialmente Consciente), prototipação, avaliação em IHC, redesign, ergonomia e usabilidade. Nesta disciplina, os alunos aprenderam técnicas e ferramentas necessárias para atividades profissionais. O professor, dessa forma, conseguiu ensinar aos estudantes como IHC é uma área central na Computação e transversal nas demais áreas, e como o conhecimento do contexto e pessoas importa no design das soluções [Pereira 2013]. As ferramentas utilizadas nesse estudo foram Cacoo para a criação de mockups e CogTool para a modelagem de tarefas. Dos 23 estudantes que responderam ao questionário online de avaliação da disciplina, 78\% ( $\mathrm{N}=18)$ informaram que voltariam a usar as ferramentas em atividades profissionais, e $22 \%(\mathrm{~N}=5)$ talvez usariam, e todos os estudantes informaram que a disciplina contribuiu para a formação e atuação profissional.

[Firmo et al. 2014] relatam também uma experiência sobre o uso de técnicas e ferramentas nos processos de ensino e aprendizagem de uma disciplina de IHC, que é obrigatória no curso de Licenciatura Plena em Computação da Universidade Federal Rural de Pernambuco (UFRPE). As atividades desta disciplina proporcionaram uma experiência prática em projeto de Design Centrado no Usuário (DCU). O objetivo de usar o DCU como metodologia alternativa na disciplina foi desenvolver nos estudantes mais responsabilidade, comprometimento e envolvimento com a disciplina, protagonismo no processo de DCU por meio da aprendizagem de técnicas de IHC na prática. Os estudantes puderam realizar pesquisas por meio de entrevistas semi-estruturadas, "teste cego" com usuários, a fim de avaliar modelos de celulares e levantar necessidades e desejos, e idear soluções por meio de análise qualitativa com o Grounded Theory [Corbin and Strauss 2014], personas, brainstorm e braindraw, prototipação por meio da ferramenta Pencil Project ${ }^{1}$ e por meio do uso de cartolina, e avaliação com usuários (Mágico de Oz e avaliação conceitual de modelos). Para registro das análises, os participantes utilizaram planilha eletrônica. Por fim, os alunos puderam colocar em prática conceitos de IHC enquanto desenvolveram novas tecnologias, trocaram e geraram ideias de forma colaborativa, e desenvolveram habilidades de autoconfiança e criatividade [Firmo et al. 2014].

Por fim, [Diniz et al. 2020] relatam um estudo realizado na disciplina de IHC em um curso tecnológico de Gestão de Tecnologia da Informação da Faculdade de Pará de Minas (FAPAM). Este estudo foi realizado de forma interdisciplinar relacionando IHC e Engenharia de Software por meio de um projeto. O objetivo do projeto era simular a integração entre as disciplinas, mostrando como essa interação acontece no mercado de trabalho. A metodologia adotada foi a baseada em projetos, de forma que estudantes construíssem um protótipo interativo de alta fidelidade de tema livre, incluindo no mínimo 4 heurísticas de usabilidade, e utilizando uma ferramenta de prototipação de livre escolha. As ferramentas utilizadas pelos alunos foram Adobe $\mathrm{XD}^{2}$ e Pencil Project de domínio de alguns alunos do $1^{\circ}$ semestre. A ferramenta Figma ${ }^{3}$ foi escolhida pelos alunos que tinham aulas de maneira remota. Os resultados das atividades práticas proporcionaram aos alunos explorarem e aplicarem suas ideias de forma criativa, praticar conceitos de IHC, prototipar colaborativamente, e praticar habilidades de resolução problemas e liderança.

Como pode ser observado, não foram encontrados na literatura estudos que relatam o uso de ferramentas como apoio aos processos de ensino e aprendizagem em discipli-

\footnotetext{
${ }^{1}$ http://pencil.evolus.vn/

${ }^{2}$ https://www.adobe.com/sea/products/xd.html

${ }^{3}$ https://www.figma.com/
} 
nas de IHC Experimental. Além disso, somente o trabalho de [Diniz et al. 2020] relatou a experiência de uma disciplina no formato remoto. Nenhum dos trabalhos cita se houve o uso da metodologia de sala de aula invertida. Desse modo, nesta artigo buscou-se descrever uma experiência sobre o uso de ferramentas utilizadas como apoio para execução de estudos primários e secundários, que aconteceu de forma remota em uma disciplina de IHC Experimental, onde buscou-se incentivar o protagonismo do estudante.

\section{Metodologia}

A disciplina de IHC Experimental teve como foco ensinar sobre planejamento, execução e análise de estudos primários e secundários. A plataforma Moodle serviu como repositório de materiais de apoio à disciplina, gerenciador das atividades por meio de instruções de execução e ambiente de entrega das atividades propostas. Além disso, a disciplina contou com o apoio de duas monitoras, que tinham o papel de apoiar as atividades dos alunos, tirar dúvidas, contribuir com a preparação de materiais para a disciplina, além de compartilhar suas experiências com os alunos. As dúvidas que surgiam durante a disciplina sobre conteúdos e atividades foram respondidas pela professora e pelas monitoras de forma assíncrona, podendo ser por meio do recurso de mensagem e fórum de discussão do $\mathrm{Mo}$ odle, ou por meio da troca de e-mails. As atividades propostas foram conduzidas a partir da dinâmica da Sala de Aula Invertida, onde os alunos precisavam realizar a leitura dos materiais que seriam discutidos posteriormente nas aulas síncronas. As aulas síncronas aconteceram de forma remota por meio da ferramenta BigBlueButton ${ }^{4}$, onde buscou-se a conversação entre discentes e docente sobre os temas trabalhados na disciplina.

De forma geral, nesta disciplina os alunos puderam exercitar seu próprio tema de pesquisa ao: (1) planejar e executar parcialmente um estudo secundário (RSL/MSL); (2) planejar um estudo primário (experimento controlado) e executar um estudo piloto deste experimento; (3) analisar quantitativamente os resultados de um experimento; (4) analisar qualitativamente os resultados de um experimento; e, (5) experimentar diferentes ferramentas e recursos de apoio à estas atividades. A disciplina foi conduzida adotando um conjunto de estratégias e de ferramentas de apoio que possibilitaram a flexibilidade da participação discente, considerando sua diversidade de condições e tempo de acesso.

No Trabalho 1 - Parte A (planejamento de um estudo secundário), os estudantes tinham que identificar e descrever: a necessidade do MSL; objetivos e questões de pesquisa; string de busca; fontes de dados (bibliotecas digitais) que utilizaram na sua busca por publicações; critérios de inclusão e exclusão adotados para seleção dos estudos; procedimentos para a seleção de artigos $\left(1^{\circ}\right.$ filtro - leitura de título e abstract e $2^{\circ}$ filtro leitura completa dos artigos); além de realizar testes e retestes na string de busca a fim de refiná-la. Para este trabalho a professora da disciplina sugeriu utilizar a ferramenta Porifera $^{5}$ como apoio a realização de algumas destas atividades.

No Trabalho 1 - Parte B (execução parcial de um estudo secundário), os estudantes tinham que: definir um formulário para extração de dados; escolher uma das bibliotecas digitais definida no planejamento; realizar a busca com a string de busca definida; considerar os 30 primeiros artigos retornados pela biblioteca digital; realizar a aplicação do $1^{\circ}$ filtro; realizar o $2^{\circ}$ filtro dos artigos que passaram no $1^{\circ}$ filtro; e extrair dados de um

\footnotetext{
${ }^{4}$ https://bigbluebutton.org/

${ }^{5}$ https://msl-research.thiagotpc.com/
} 
dos estudos que passou no $2^{\circ}$ filtro. A ferramenta Porifera também foi sugerida para esta parte do Trabalho 1.

No Trabalho 2 (planejamento de um experimento controlado e execução de um teste piloto deste experimento) os alunos tiveram que: definir os objetivos do experimento (de acordo com o paradigma GQM - (Goal-Question-Metric)), proposto por [Basili and Rombach 1988]; formular as hipóteses nula e alternativa; selecionar as variáveis dependente e independentes; definir como as variáveis dependentes serão coletadas e/ou calculadas; especificar o design do estudo: between group ou within group, conforme [Lazar et al. 2017]; realizar a seleção de participantes; definir o ambiente onde o estudo será realizado; definir os instrumentos utilizados no experimento; avaliar as ameaças à validade; e executar um teste piloto com pelo menos duas pessoas. Para este trabalho, foram sugeridas ferramentas de edição de texto.

No Trabalho 3 (análise quantitativa de um experimento) o estudante tinha que identificar um artigo científico dentro do seu tema de pesquisa, que descrevesse um experimento similar ao que foi planejado no Trabalho 2, e que continham os dados brutos que pudessem ser reproduzidos por meio de testes estatísticos. Além disso, os alunos precisavam utilizar um software estatístico. Foram sugeridos o SPSS ${ }^{6}$ ou R ${ }^{7}$.

No Trabalho 4 (análise qualitativa de um experimento) o estudante tinha que: escolher dados qualitativos obtidos através de algum experimento relacionado ao seu tema pesquisa; analisar e codificar os dados qualitativos, identificando, nomeando e registrando conteúdos recorrentes no conjunto completo dos dados (codificação aberta); examinar os códigos obtidos e encontrar categorias mais abstratas em que os códigos da etapa anterior poderiam ser agrupados (codificação axial). Foi sugerida a ferramenta Atlas.ti ${ }^{8}$.

Cada trabalho teve a duração de 1 semana. O Trabalho 1 (parte A e B) foi realizado individualmente e os demais trabalhos em dupla. A entrega de cada trabalho foi um relatório contendo a descrição de cada item solicitado. Vale ressaltar que para cada trabalho foram sugeridas ferramentas, porém, os alunos tinham a liberdade de utilizar outra de sua preferência. Além disso, os alunos tiveram a liberdade de utilizar o seu tema de pesquisa para contextualizar as atividades da disciplina. Ao final da disciplina, estudantes responderam um questionário com 07 questões para relatar suas experiências com as ferramentas utilizadas. Os dados resultantes das questões de 1 à 5 foram analisados quantitativamente, e os das questões 6 e 7 foram analisados qualitativamente. Os resultados de cada análise, respectivamente, são apresentados nas seções a seguir.

\section{Análise Quantitativa}

Nesta seção são apresentados os resultados da análise quantitativa sobre as ferramentas de apoio a pesquisa experimental utilizadas na disciplina de IHC Experimental. Os dados para a análise foram coletados a partir de 05 questões de múltipla escolha, em que estudantes relataram sobre as ferramentas utilizadas em cada trabalho: sendo a questão 1 sobre Trabalho 1 (Parte A e B); a questão 2 sobre o Trabalho 2; a questão 3 sobre o Trabalho 3; a questão 4 sobre o Trabalho 4; e a questão 5 perguntava ao estudante qual das ferramentas utilizou na disciplina mas que não conhecia anteriormente.

\footnotetext{
${ }^{6}$ https://www.ibm.com/br-pt/analytics/spss-statistics-software

${ }^{7}$ https://www.r-project.org/

${ }^{8}$ https://atlasti.com/
} 
A Figura 1 apresenta uma visão geral das ferramentas utilizadas durante a execução das atividades propostas na disciplina. Pode-se observar que a soma das porcentagens passam de $100 \%$, pois os alunos puderam utilizar mais de uma ferramenta durante a execução das atividades. A Figura 1A está relacionada às ferramentas utilizadas na execução do estudo secundário (Trabalho 1). É possível observar que $100 \%(\mathrm{~N}=13)$ dos estudantes responderam que usaram a ferramenta Porifera. Isto porque os alunos foram instruídos a usar esta ferramenta, que é colaborativa para conduzir RSL/MSL, para que também as monitoras pudessem auxiliar neste trabalho. Em seguida, pode ser observado que $46,15 \%(\mathrm{~N}=6)$ responderam que fizeram uso de ferramentas de edição de texto, seguido de 23,08\% ( $\mathrm{N}=3)$ que fizeram uso de ferramentas de edição de planilha. Esse resultado pode estar relacionado ao uso de ferramentas de apoio ao planejamento de uma RSL/MSL.

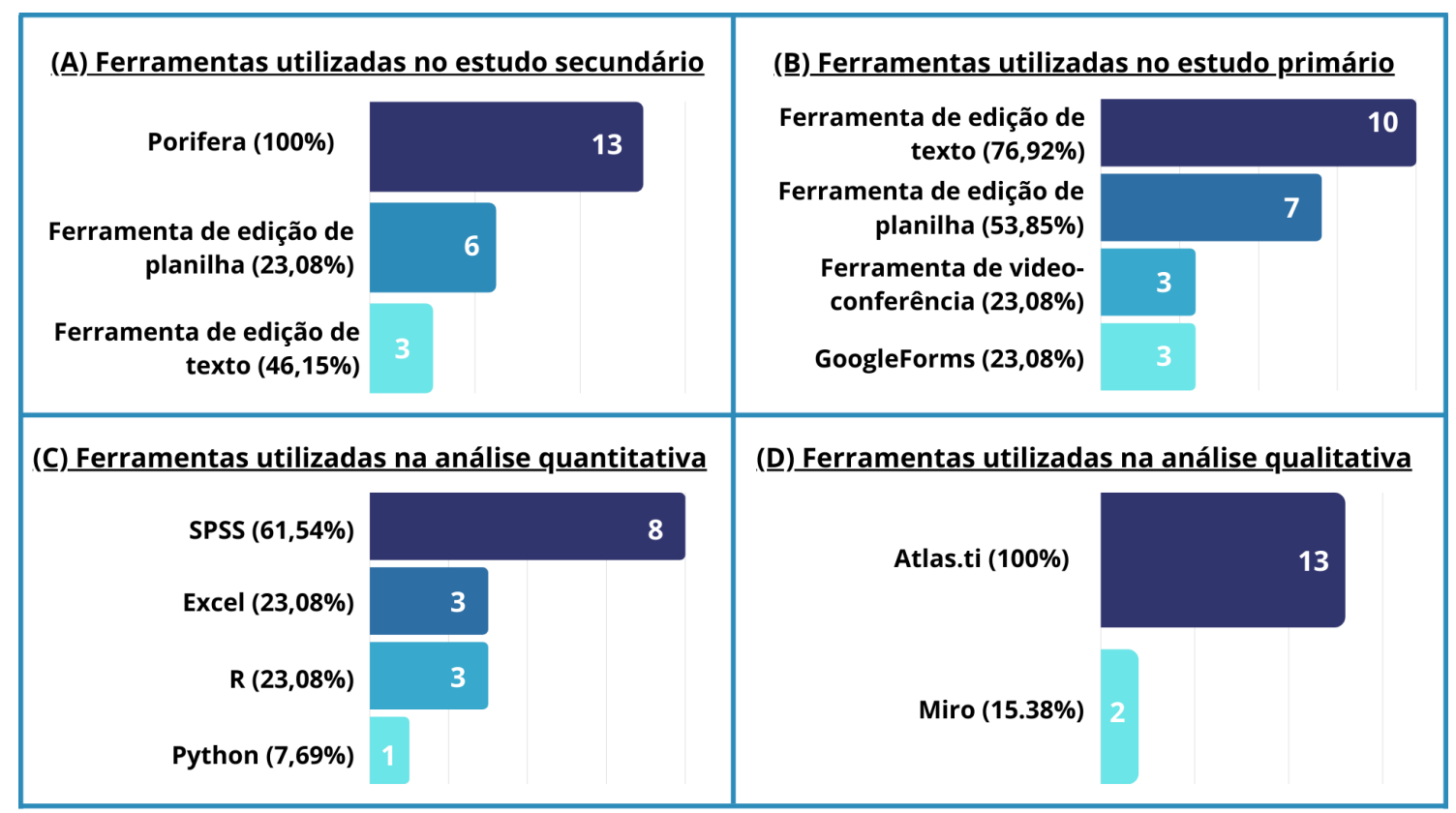

Figura 1. Ferramentas utilizadas nos trabalhos da disciplina.

A Figura 1B apresenta os resultados relacionado às ferramentas utilizadas para executar a atividade que consiste em um estudo primário (Trabalho 2). Para essa atividade, $76,92 \%(\mathrm{~N}=10)$ dos estudantes responderam que utilizaram ferramentas de edição de texto; 53,85\% ( $\mathrm{N}=7$ ) ferramentas de edição de planilha, seguido de 23,08\% ( $\mathrm{N}=$ 3) que utilizaram a ferramenta Google Forms. Por fim, 23,08\% $(\mathrm{N}=3)$ responderam ter utilizado ferramentas de videoconferência. O grande uso de ferramentas de edição de texto e planilha é referente ao desenvolvimento dos materiais utilizados durante o planejamento do experimento e para a escrita do relatório. Além disso, nesta atividade, os alunos puderam conduzir o estudo piloto do experimento com seus participantes de maneira remota por videoconferência ou presencial com familiares da mesma casa. Por isso, alguns alunos $(\mathrm{N}=3)$ utilizaram ferramenta de videoconferência. A ferramenta de formulário GoogleForms também foi utilizada para a execução do teste piloto do experimento, para que os alunos pudessem obter ofeedback dos participantes do seu piloto.

A Figura $1 \mathrm{C}$ apresenta os resultados relacionados às ferramentas utilizadas para realizar a atividade de análise quantitativa (Trabalho 3). É possível observar que 61,54\% 
$(\mathrm{N}=8)$ dos estudantes utilizaram o SPSS, 23,08\% $(\mathrm{N}=3)$ responderam ter utilizado o Excel, seguido de 23,08\% ( $\mathrm{N}=3)$ que utilizaram a ferramenta $\mathrm{R}$. Por fim, 7,69\% (N=1) respondeu ter utilizado a linguagem de programação Python. A ferramenta mais utilizada foi o SPSS, pois os alunos tinham mais materiais de apoio para o uso dessa ferramenta. Além disso, os alunos optaram pelo uso de outras ferramentas conhecidas para análise estatística como R e Excel. Um dos estudantes utilizou a linguagem de programação Python devido a sua familiaridade com esta linguagem para fazer este tipo de análise.

A Figura 1D apresenta os resultados relacionados às ferramentas utilizadas durante a atividade de análise qualitativa (Trabalho 4). Dos 13 estudantes da disciplina, $100 \%$ responderam ter utilizado o Atlas.ti, seguido de $15,38 \%(\mathrm{~N}=2)$ que responderam ter utilizado a ferramenta Miro ${ }^{9}$. O Atlas.ti também foi indicado durante a aula, o que implicou no alto uso por estudantes para realizar a análise qualitativa dos dados. Dois alunos, além de utilizarem o Atlas.ti também utilizaram o Miro, uma ferramenta que permite uma melhor visualização de informações de forma colaborativa.

Já a Figura 2 apresenta os resultados relacionados às ferramentas que os estudantes não conheciam antes da disciplina. Pode ser observado que $100 \%(\mathrm{~N}=13)$ dos estudantes responderam que não conheciam a ferramenta Atlas.ti, 84,62\% $(\mathrm{N}=11)$ não conheciam a ferramenta Porifera, 53,85\% ( $\mathrm{N}=7)$ não conheciam o SPSS e 7,69\% ( $\mathrm{N}=1)$ não conhecia a ferramenta $\mathrm{R}$. Esses resultados podem indicar que as sugestões de ferramentas dadas pela professora são importantes, pois permitem que os alunos conheçam novas ferramentas e possam utilizá-las tanto em seu contexto acadêmico como profissional.

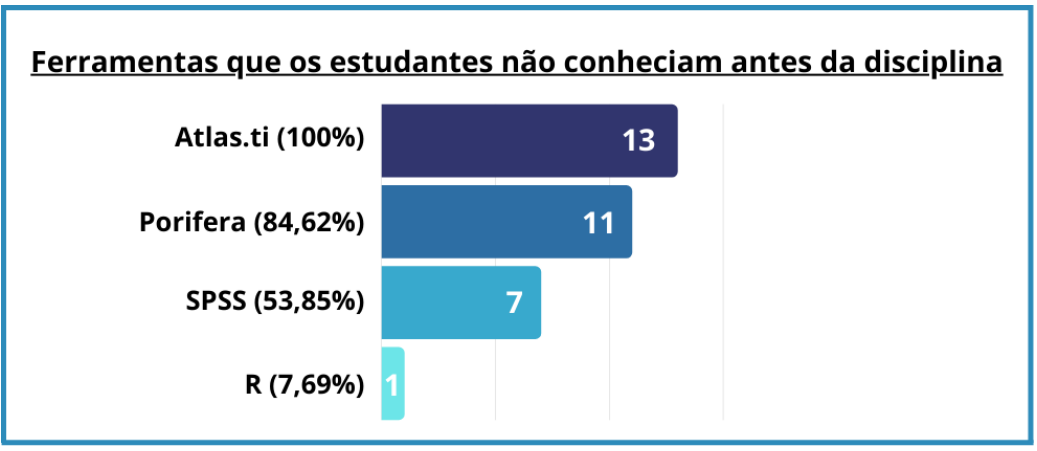

Figura 2. Ferramentas que os estudantes não conheciam antes da disciplina.

\section{Análise Qualitativa e Discussões}

Nesta seção são apresentados os resultados obtidos da análise qualitativa das questões abertas sobre as ferramentas de apoio a pesquisa experimental. As questões foram: (1) "Quais dificuldades/limitações você enfrentou para utilizar estas ferramentas na disciplina?" e (2) "Alguma destas ferramentas não atendeu sua expectativa durante a atividade da disciplina? Se sim, qual ferramenta e em que ela não atendeu?". Para análise dos dados obtidos nestas questões, utilizou-se um subconjunto dos procedimentos do método Grounded Theory (GT), definido por [Corbin and Strauss 2014]. O GT possui 3 etapas no processo de codificação, que são codificação aberta, axial e seletiva. Na codificação aberta codificou-se a resposta dada por cada participante nas 2 questões citadas acima;

\footnotetext{
${ }^{9}$ Miro: https://miro.com/
} 
e na codificação axial os códigos foram agrupados de acordo com suas propriedades e relacionados entre si, formando categorias que representavam suas características. A codificação seletiva não foi realizada, pois não se pretende criar uma teoria. As etapas de codificação aberta e axial foram suficientes para compreender as dificuldades enfrentadas pelos alunos ao utilizar as ferramentas durante a execução das atividades propostas. Para a apresentação dos resultados, cada estudante recebeu um identificador com P (de participante) mais um número (de 1 à 13), que serão discutidos a seguir.

\subsection{Facilidade no uso das ferramentas}

Mesmo que as duas questões abertas não tivessem perguntas sobre a facilidade no uso das ferramentas, alguns participantes relataram sobre isso. De modo geral, os participantes consideraram que as ferramentas foram satisfatórias para a realização dos trabalhos da disciplina (veja a citação de P12 abaixo); são intuitivas e fáceis de usar (veja as citações de P3 e P2 abaixo); foram úteis e atenderam às expectativas (veja as citações de P5, P7 e P9 abaixo).

"As ferramentas foram satisfatórias para a realização dos trabalhos (P12)".

"Não houveram dificuldades, são intuitivas e fáceis de usar (P3)”.

“As ferramentas são de fácil manuseio (P2)”.

“Todas atenderam as expectativas, foram muito úteis (P5)”.

"No geral, todas elas atenderam o que foi solicitado durante a disciplina para sua ехесис̧а̃o (P7)”.

"Creio que todas as ferramentas cumpriram com minhas expectativas (P9)"

Os dados qualitativos demonstram que as ferramentas utilizadas para a execução das atividades são fáceis de serem utilizadas. Além disso, foram consideradas intuitivas. Esse resultado pode ter influenciado para que os alunos tenham se sentido satisfeitos ao utilizá-las, considerando também o atendimento às expectativas. Isso implica dizer que as ferramentas utilizadas apoiaram as atividades de planejamento, execução e análise dos estudos realizados na disciplina.

\subsection{Dificuldade no uso da ferramenta}

As dificuldades de uso foram relatadas em relação às ferramentas Atlas.ti, SPSS e Porifera utilizadas na disciplina. Sobre o Atlas.ti, os estudantes relataram que esta ferramenta em sua versão web não possibilita trabalhar de forma colaborativa (veja a citação de P8 abaixo), é difícil criar as categorias que ligam os códigos (veja a citação de P10 abaixo), e é considerada pouco intuitiva (veja a citação de P11 abaixo).

"O Atlas.ti tem uma versão web, mas não me permite convidar alguém para colaborar, só se eu baixar a versão desktop, porém eles não oferecem uma versão para Linux (P8)".

“Atlas.ti - tive dificuldade em criar os networks que ligavam os codes (P10)”.

"O Atlas.ti foi a ferramenta utilizada que considerei menos intuitiva. Mas mesmo assim consegui realizar as atividades esperadas (P11)”. 
Sobre o SPSS, os estudantes destacaram as muitas funcionalidades e opções, dificultando encontrar o que se deseja na ferramenta (veja a citação de P3 abaixo) e o material de ajuda parece ser antigo (veja a citação de P4 abaixo).

"SPSS tem muitas funcionalidades, muitas opções, isto dificulta um pouco encontrar o que se deseja (P3)".

\section{“Conteúdo sobre SPSS parecia um pouco antigo (P4)".}

Quanto a ferramenta Porifera, a dificuldade foi relacionada à importação das bibliotecas nesta ferramenta (veja a citação de P7 abaixo).

"Uma dificuldade que possuí foi na importação das bibliotecas para a plataforma Porifera. Conversei com [o autor da ferramenta] e dentro de dias resolvemos, porém fiquei limitada para seguir com a atividade e o cronograma a qual havia me organizado (P7)”.

De modo geral, as dificuldades sobre o Atlas.ti estão relacionadas a não possibilidade de trabalhar de forma colaborativa devido a limitação da ferramenta em sua versão web. Além disso, esta ferramenta não disponibiliza versão desktop para sistema operacional GNU/Linux. O relato dessa dificuldade enfrentada por P8 traz uma reflexão sobre sugerir ferramentas na disciplina para análise qualitativa que permitam trabalhar de forma colaborativa. Além disso, em relação à dificuldade em criar as categorias nesta ferramenta, os alunos poderiam procurar material de apoio ou retirar dúvidas com a professora e monitoras. Já em relação à ferramenta SPSS, as dificuldades são mais direcionadas a materiais de apoio antigo e dificuldade de usá-la devido às diversas funcionalidades que a mesma apresenta. Vale ressaltar que os alunos tinham liberdade para utilizarem outras ferramentas. Porém, fica como reflexão sugerir ferramentas mais intuitivas e de fácil uso, e que inclua materiais de apoio mais atuais nas próximas turmas desta disciplina. Por fim, houve um relato sobre a ferramenta Porifera em relação à dificuldade de importação das publicações identificadas nas bibliotecas nesta ferramenta. Esta dificuldade foi sanada entrando em contato com o autor da ferramenta. Uma forma de minimizar as dificuldades enfrentadas no uso das ferramentas foi por meio da disponibilização de materiais auxiliares e retirada de dúvidas com a professora e monitoras, sendo fundamental para que eles pudessem prosseguir com as atividades.

\subsection{Dificuldade na aprendizagem da ferramenta}

As dificuldades na aprendizagem das ferramentas estão relacionadas a barreira na curva de aprendizagem (ver citações de P1 e P12 abaixo) ou não entender como a ferramenta funciona (P13).

"De barreira apenas a curva de aprendizagem de cada ferramenta (P1)”.

"A princípio não foram percebidas grandes dificuldades no manuseio dos softwares, apenas a curva de aprendizado natural previsivel quando se tem contato com ferramentas novas (P12)”.

"SPSS - não entendi se ela precisa de muitos dados brutos para funcionar, acabei não conseguindo fazer meu trabalho direito por conta disso, pois o único artigo que achei com dados quantitativos, só possuíam 2 linhas de dados (P13)”.

As dificuldades quanto a curva de aprendizagem, apontadas por P1 e P12, e a falta 
de entendimento sobre como usar, como apresentado por P13, podem estar associadas aos primeiros usos de uma ferramenta não conhecida e a falta de experiência com a atividade solicitada. Dessa forma, é essencial que pessoas mais experientes, como docente e monitor estejam disponíveis para auxiliar estudantes no decorrer das atividades, para que questões técnicas não impeçam a geração de novos conhecimentos. Além disso, como a disciplina seguiu uma metodologia de Sala de Aula Invertida, os alunos foram incentivados a serem protagonistas do seu aprendizado, buscando meios e materiais para sanarem suas dúvidas, tais como video-aulas, sites e tutoriais sobre as ferramentas.

\section{Considerações Finais e Trabalhos Futuros}

O objetivo deste relato de experiência foi analisar ferramentas de apoio a pesquisa experimental em uma disciplina de IHC Experimental. O uso das ferramentas se mostrou eficaz no aprendizado dos estudantes da disciplina. Isso pode ser observado através dos resultados qualitativos, que mostram que algumas ferramentas atenderam às expectativas, são fáceis de usar e úteis. No entanto, algumas dificuldades pontuais também foram percebidas nas ferramentas utilizadas. Estas dificuldades podem ser enfrentadas lendo materiais de apoio, estudando mais sobre as ferramentas e entrando em contato com pesquisadores que conhecem o uso destas ferramentas, tais como orientador, professor e monitor. Os dados quantitativos demonstram a importância do uso das ferramentas sugeridas durante as atividades, já que a maioria dos alunos não conhecia estas ferramentas. Estas sugestões oportunizaram aos alunos conhecer, estudar e aplicar estas ferramentas nas atividades da disciplina. Além disso, eles poderão utilizá-las em pesquisas futuras.

Este artigo contribui para o avanço da Educação em Computação principalmente sobre disciplinas no âmbito de pesquisa experimental. Com este artigo é possível compreender que o uso de ferramentas em disciplinas voltadas para pesquisa experimental pode desenvolver nos estudantes experiências práticas de pesquisa, além de apoiá-los na realização de estudos. Como trabalhos futuros, pretende-se sugerir outras ferramentas durante a disciplina de IHC Experimental que atenda as necessidades apontadas neste estudo. Percebeu-se que uma das características importantes para a ferramenta ser utilizada neste tipo de disciplina é a colaboratividade, já que os trabalhos normalmente são realizados em duplas ou equipes. A partir da definição de novas ferramentas para esta disciplina, pretende-se obter o feedback dos alunos das próximas turmas a fim de observar se as necessidades identificadas no presente estudo foram resolvidas.

\section{Referências}

Basili, V. R. and Rombach, H. D. (1988). Towards a comprehensive framework for reuse: A reuse-enabling software evolution environment.

Corbin, J. and Strauss, A. (2014). Basics of qualitative research: Techniques and procedures for developing grounded theory. Sage publications.

Diniz, L. M. F., Pereira, M. V., de Paula, L. J. D., and de Melo Silva, E. C. (2020). Aprendizado baseado em projetos em ihc (presencial e remoto): prototipação segundo as heurísticas de nielsen. In Anais Estendidos do XIX Simpósio Brasileiro de Fatores Humanos em Sistemas Computacionais, pages 13-18. SBC. 
Firmo, D., Gondim, H., and Falcão, T. (2014). Alunos como protagonistas na construção do conhecimento: uma experiência prática na disciplina de ihc. In Anais do XXII Workshop sobre Educação em Computação, pages 279-288. SBC.

Kitchenham, B. and Charters, S. (2007). Guidelines for performing systematic literature reviews in software engineering.

Lazar, J., Feng, J. H., and Hochheiser, H. (2017). Research methods in human-computer interaction. Morgan Kaufmann.

Pereira, R. (2013). Ihc na especialização em engenharia de software: discussões sobre uma experiência prática. In WEIHC, pages 1-6.

Valente, J. A. (2014). Blended learning e as mudanças no ensino superior: a proposta da sala de aula invertida. Educar em revista, pages 79-97. 